Supporting Information for

\title{
Prediction of Highly Selective Electrocatalytic Nitrogen Reduction at Low Overpotential on a Mo-doped g-GaN Monolayer
}

Lesheng Li, ${ }^{\dagger}$ J. Mark P. Martirez ${ }^{\ddagger}$, and Emily A. Carter ${ }^{*},+\neq, \|$

${ }^{\dagger}$ Department of Mechanical and Aerospace Engineering, Princeton University, Princeton, NJ 08544-5263, United States and ${ }^{\ddagger}$ Department of Chemical and Biomolecular Engineering, and "Office of the Chancellor, Box 951405, University of California, Los Angeles, Los Angeles, CA 90095-1405, United States

* To whom correspondence should be addressed. Email: eac@ucla.edu (EAC) 
Table S1. Adsorption energies (eV) for $\mathrm{N}_{2}$ adsorption on the Mo@g-GaN monolayer using three different Mo PBE PAW potentials with different numbers of electrons solved for selfconsistently, using an implicit solvation model and DFT-PBE-D3.*

\begin{tabular}{|c|c|c|c|c|}
\hline \multirow{2}{*}{$\begin{array}{l}\text { Self-consistent Mo } \\
\text { electron configuration }\end{array}$} & & \multicolumn{3}{|c|}{ Energy [eV] } \\
\hline & & $\mathrm{E}\left(\mathrm{N}_{2}+\mathrm{Mo} @ \mathrm{~g}-\mathrm{GaN}\right)(M)^{* *}$ & $\begin{array}{c}\mathrm{E}(\mathrm{Mo@g-GaN}) \\
(M)^{* *}\end{array}$ & $\mathrm{E}_{\text {ads }}\left(\mathrm{N}_{2}\right)$ \\
\hline \multirow{8}{*}{$4 d^{5} 5 s^{1}$} & \multirow{4}{*}{ End-on } & $-205.29(M=0)$ & \multirow{8}{*}{$\begin{array}{l}-188.65(M=0) \\
-188.53(M=1) \\
-187.99(M=3)\end{array}$} & \multirow{4}{*}{-0.66} \\
\hline & & $-205.39(M=1)$ & & \\
\hline & & $-205.40(M=1.3)$ & & \\
\hline & & $-205.06(M=3)$ & & \\
\hline & \multirow{4}{*}{ Side-on } & $-205.02(M=0)$ & & \multirow{4}{*}{-0.39} \\
\hline & & $-205.14(M=0.87)$ & & \\
\hline & & $-205.13(M=1)$ & & \\
\hline & & $-204.12(M=3)$ & & \\
\hline \multirow{8}{*}{$4 p^{6} 4 d^{5} 5 s^{1}$} & \multirow{4}{*}{ End-on } & $-205.53(M=0)$ & \multirow{8}{*}{$\begin{array}{l}-\mathbf{1 8 8 . 4 2}(M=\mathbf{0}) \\
-188.28(M=1) \\
-187.84(M=3)\end{array}$} & \multirow{4}{*}{-0.60} \\
\hline & & $-205.62(M=1)$ & & \\
\hline & & $-205.64(M=1.37)$ & & \\
\hline & & $-205.32(M=3)$ & & \\
\hline & \multirow{4}{*}{ Side-on } & $-205.28(M=0)$ & & \multirow{4}{*}{-0.32} \\
\hline & & $-205.35(M=0.78)$ & & \\
\hline & & $-205.34(M=1)$ & & \\
\hline & & $-204.31(M=3)$ & & \\
\hline \multirow{8}{*}{$4 s^{2} 4 p^{6} 4 d^{5} 5 s^{1}$} & & $-205.52(M=0)$ & \multirow{8}{*}{$\begin{array}{l}-188.41(M=0) \\
-188.27(M=1) \\
-187.83(M=3)\end{array}$} & \multirow{4}{*}{-0.59} \\
\hline & & $-205.61(M=1)$ & & \\
\hline & End-on & $-205.63(M=1.36)$ & & \\
\hline & & $-205.31(M=3)$ & & \\
\hline & \multirow{4}{*}{ Side-on } & $-205.27(M=0)$ & & \multirow{4}{*}{-0.31} \\
\hline & & $-205.35(M=0.78)$ & & \\
\hline & & $-205.34(M=1)$ & & \\
\hline & & $-204.31(M=3)$ & & \\
\hline
\end{tabular}

* Shown in bold are the ground-state total energies and magnetizations for Mo@g-GaN and $\mathrm{N}_{2}+\mathrm{Mo} @ g-G a N$ for each PAW potential.

${ }^{* *} M$ in parentheses indicates the total magnetization of the entire system. $M$ was constrained initially to 0,1 , and 3 to help determine eventually the ground-state magnetization. These values were chosen based on the expected spins of Mo, which alone can give rise to magnetization, namely, low-spin $\mathrm{Mo}(\mathrm{IV})$ and $\mathrm{Mo}(\mathrm{VI}): S_{z}=0$, and low- and high-spin $\mathrm{Mo}(\mathrm{III}): S_{z}=1 / 2$ and 3/2. Note that the adsorbate-free Mo@g-GaN favors $M=0$, despite having an odd number of electrons in the unit cell. The metallic character of the monolayer gives rise to fractional occupation around the Fermi level that leads to this behavior. 

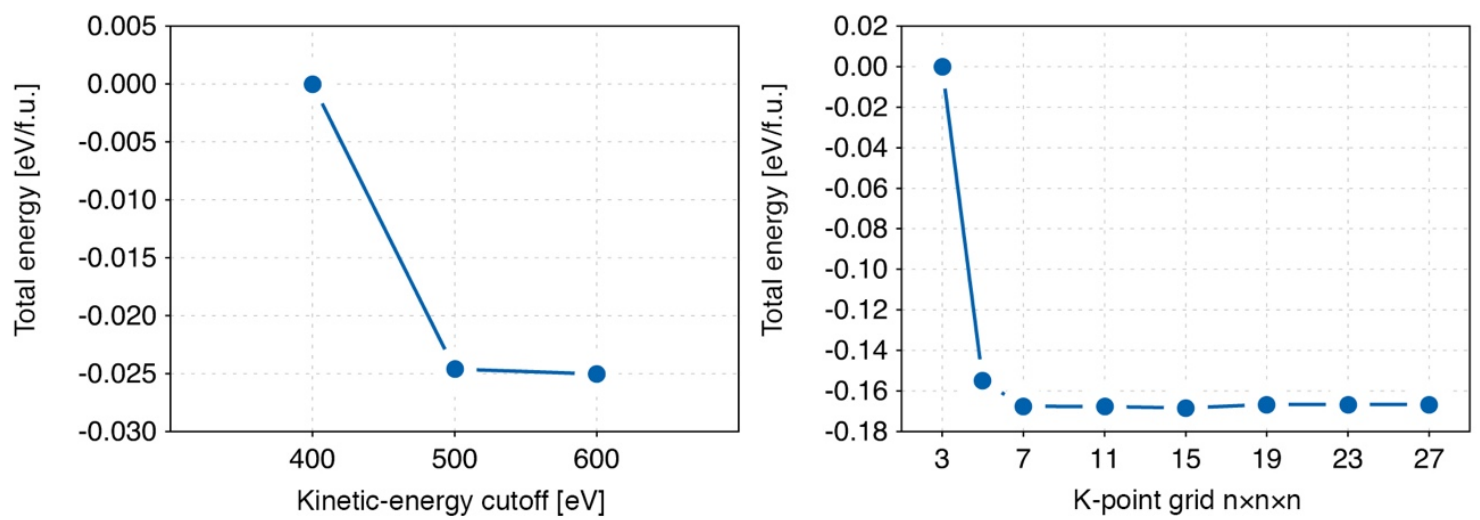

Figure S1. Convergence tests of the plane-wave basis kinetic-energy cutoff and k-point grid for the total energy (eV/formula-unit) of the two-atom wurtzite GaN primitive cell $(a=b=3.20 \AA$, $\left.\mathrm{c}=5.21 \AA, \alpha=\beta=90^{\circ}, \gamma=120^{\circ}\right)$. Convergence at the chosen parameters $(500 \mathrm{eV}$ cutoff and $19 \times 19 \times 19$ $\mathrm{k}$-point grid) is within $0.5 \mathrm{meV} / \mathrm{atom}$.

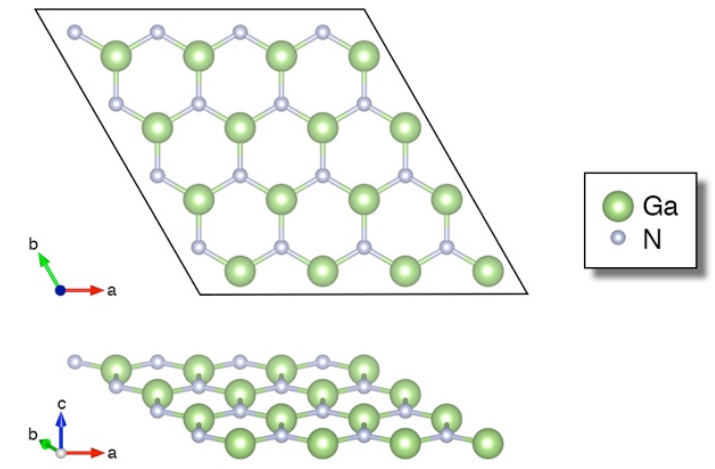

Figure S2. Top and side views of the optimized geometry of the pristine GaN monolayer, based on DFT-PBE-D3 calculations using an implicit solvation model. 

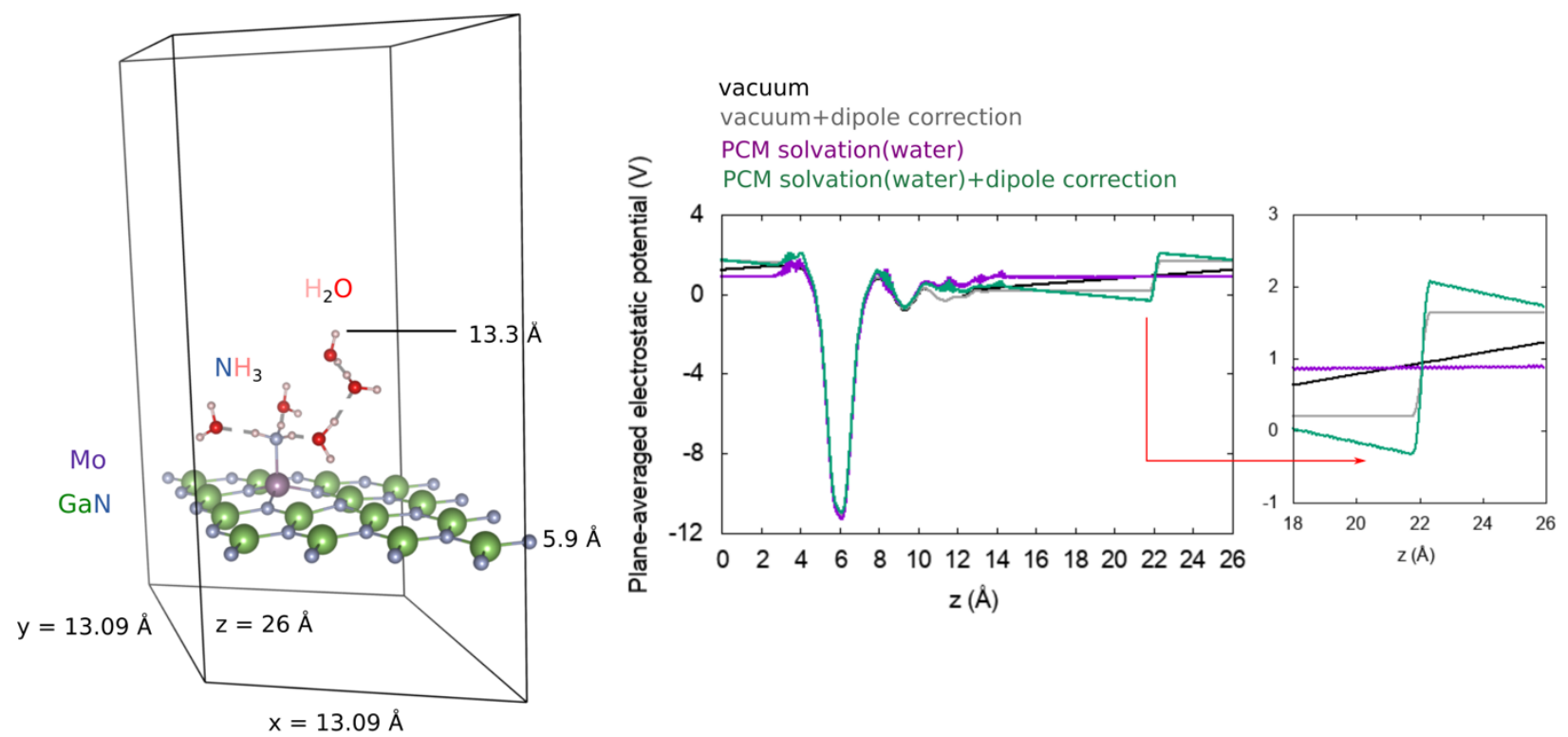

Figure S3. Center panel: plane-averaged electrostatic potential along the surface normal for the ${ }^{*} \mathrm{NH}_{3}$ intermediate on the Mo@g-GaN with explicit solvating water molecules (structure shown on the left panel) when in vacuum (black), vacuum + dipole correction (energy and potential corrected, ${ }^{1-3}$ gray), linearized Poisson-Boltzmann polarizable continuum model (PCM ${ }^{4-5}$ for implicit solvation (purple), and PCM + dipole correction (green). Right panel: zoomed-in portion roughly in the middle of the vacuum emphasizing the convergence behavior of the "vacuum" potentials.

To examine the necessity of including a dipole correction along the surface normal, we performed four test calculations of adsorbed ammonia $\left({ }^{*} \mathrm{NH}_{3}\right)$ on Mo@g-GaN with five explicit solvating water molecules in: (1) vacuum, (2) vacuum + a dipole correction (energy and potential corrected), ${ }^{1-3}$ (3) implicit solvation (via the linearized Poisson-Boltzmann polarizable continuum model), ${ }^{4-5}$ and (4) implicit solvation + a dipole correction (energy and potential corrected). As seen in Figure S3, the mixed implicit + explicit solvation model without dipole correction (purple in Figure S3) correctly exhibits a flat (converged) electrostatic potential in the vacuum region. This is because the implicit solvation model already screens the surface dipole due to its dielectric response. In fact, the dipole correction appears incompatible with implicit solvation as it leads to a non-convergent vacuum potential (green in Figure S3). On the other hand, the vacuum only 
calculation exhibits a sloped electrostatic potential (black in Figure S3) as expected, while applying the dipole correction alone correctly flattens the potential on both sides of the slab with a discontinuity (step) approximately in the middle of the vacuum region (gray in Figure S3). The aforementioned potential step arises due to the asymmetric arrangement of the adsorbate and explicit solvating water molecules. Therefore, a dipole correction is not employed when implicit solvation is used.

Table S2. Deviation of the lattice constant $a$ of the TM@g-GaN monolayers compared to that in the pristine GaN monolayer, based on DFT-PBE-D3 calculations with implicit solvation.

\begin{tabular}{cccccc}
\hline \hline TM atom & $\mathrm{Sc}$ & $\mathrm{Ti}$ & $\mathrm{V}$ & $\mathrm{Cr}$ & $\mathrm{Mn}$ \\
\hline$\Delta a[\%]$ & 1.82 & 1.53 & 1.46 & 1.22 & 1.32 \\
\hline \hline TM atom & $\mathrm{Fe}$ & $\mathrm{Co}$ & $\mathrm{Ni}$ & $\mathrm{Cu}$ & $\mathrm{Zn}$ \\
\hline$\Delta a[\%]$ & 1.37 & 1.21 & 1.18 & 1.28 & 1.54 \\
\hline \hline TM atom & $\mathrm{Mo}$ & $\mathrm{Ru}$ & $\mathrm{Rh}$ & $\mathrm{Pd}$ & $\mathrm{Ag}$ \\
\hline$\Delta a[\%]$ & 1.89 & 1.62 & 1.39 & 1.53 & 1.82 \\
\hline \hline
\end{tabular}

Table S3. Finite size error introduced in the $4 \times 4$ supercell modelling a Mo@g-GaN monolayer, based on DFT-PBE-D3 calculations with implicit solvation.

\begin{tabular}{|c|c|c|}
\hline \multirow{2}{*}{ Species } & \multicolumn{2}{|c|}{$\mathrm{E}_{\mathrm{ads}}[\mathrm{eV}]$} \\
\hline & $4 \times 4$ supercell & $5 \times 5$ supercell \\
\hline \multirow{2}{*}{${ }^{*} \mathrm{~N}_{2}$} & -0.66 (end-on) & -0.64 (end-on) \\
\hline & -0.39 (side-on) & -0.38 (side-on) \\
\hline \multirow{2}{*}{${ }^{*} \mathrm{NNH}$} & -3.97 (end-on) & -3.95 (end-on) \\
\hline & -3.15 (side-on) & -3.14 (side-on) \\
\hline${ }^{*} \mathrm{NH}_{2}$ & -3.51 & -3.49 \\
\hline
\end{tabular}


(a)

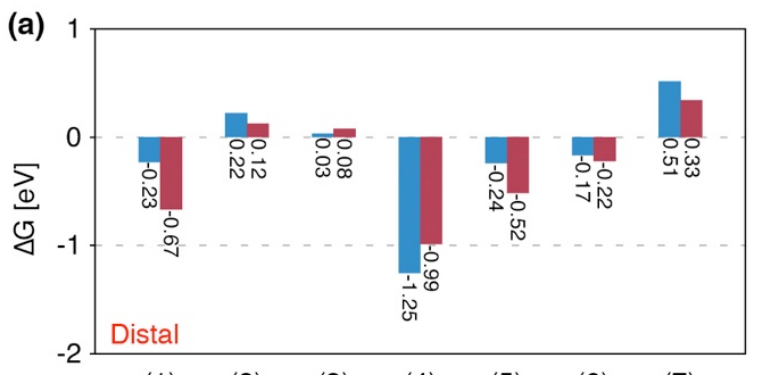

$\begin{array}{lllllll}(1) & (2) & \text { (3) } & \text { (4) } & \text { (5) } & \text { (6) } & \text { (7) }\end{array}$

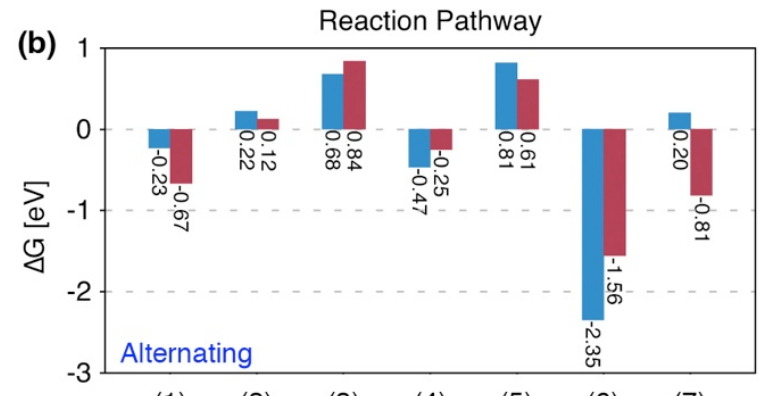

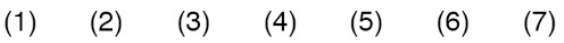

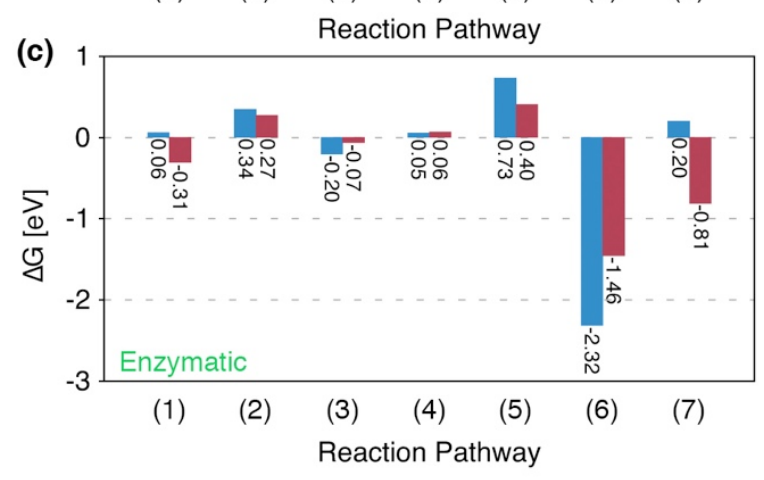

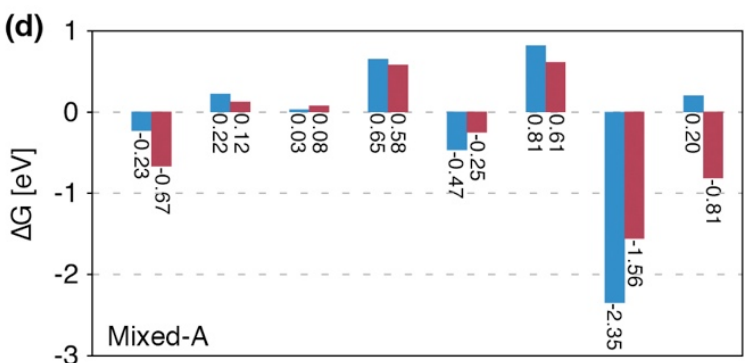

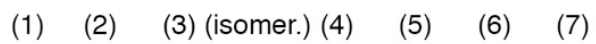

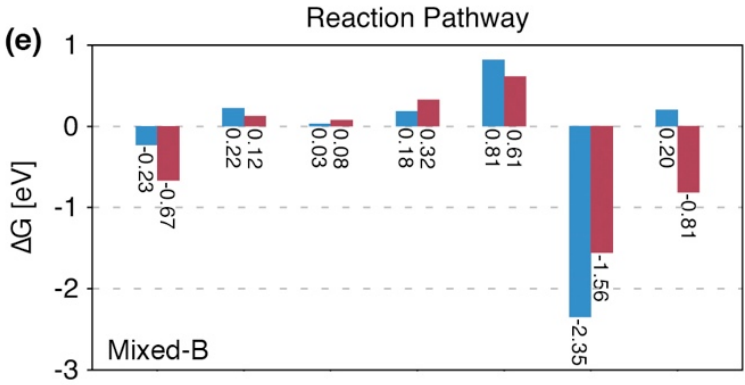

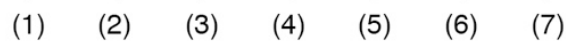

(f)

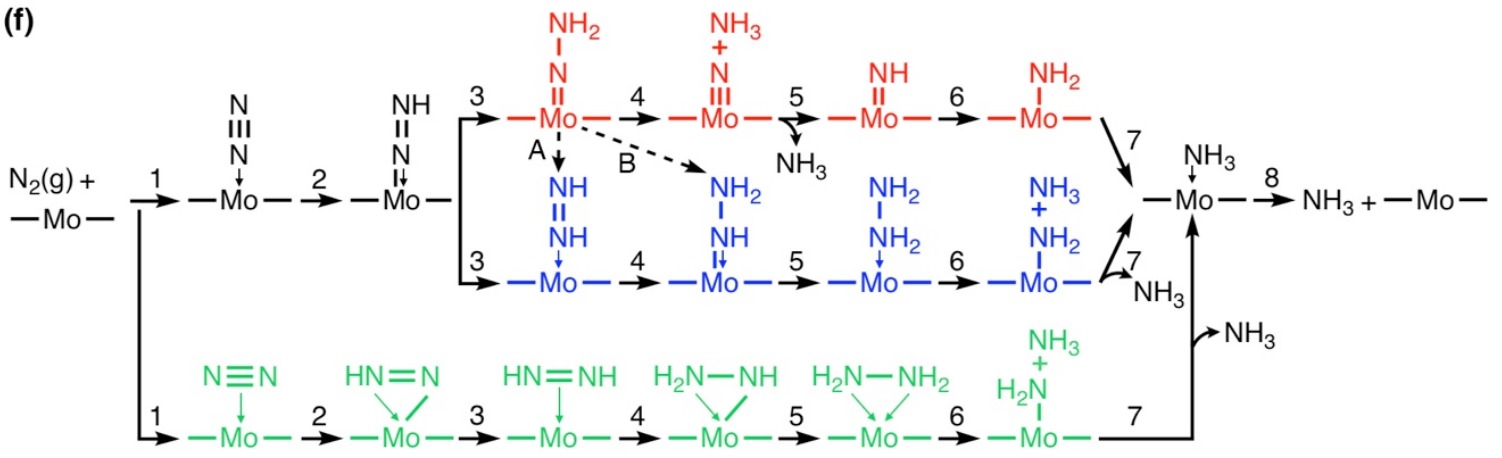

Figure S4. Reaction Gibbs free energy of each NRR elementary step using either an implicit solvation model (blue) or a mixed implicit + explicit solvation model (red), along the (a) distal, (b) alternating, (c) enzymatic, (d) mixed-A, and (e) mixed-B mechanisms, based on DFT-PBE-D3 calculations. Step (isomer.) in (d) is the ${ }^{*} \mathrm{NNH}_{2}$ to ${ }^{*} \mathrm{NHNH}$ isomerization step (labeled A in (f)) in the mixed-A pathway. Numerical values are shown at the end of each bar. The step indices on the horizontal axis of (a)-(e) correspond to the step numbers in (f). 
Calculated reaction Gibbs free energies of each NRR elementary step are displayed in Figure S4, where predictions based on implicit solvation and implicit + explicit solvation models are shown in blue and red, respectively. Steps 1-7 on the horizontal axis of Figure S4a-e correspond to those shown in Figure S4f. As can be seen in Figure S4, the predicted PDSs are the same in both solvation models for all mechanisms except the alternating one (vide infra): ${ }^{*} \mathrm{NH}_{2}$ to ${ }^{*} \mathrm{NH}_{3}$ in distal (step 7, Figure S4a), $\mathrm{HN}^{*}-{ }^{*} \mathrm{NH}_{2}$ to $\mathrm{H}_{2} \mathrm{~N}^{*}-{ }^{*} \mathrm{NH}_{2}$ in enzymatic (step 5, Figure S4c), ${ }^{*} \mathrm{NHNH}_{2}$ to ${ }^{*} \mathrm{NH}_{2} \mathrm{NH}_{2}$ in mixed-A (step 5, Figure S4d) and mixed-B (step 5, Figure S4e). The reaction Gibbs free energy of the PDS based on the implicit and the implicit + explicit solvation calculations are 0.51 vs. $0.33 \mathrm{eV}, 0.73$ vs. $0.40 \mathrm{eV}$, and 0.81 vs. $0.61 \mathrm{eV}$ for distal, enzymatic, and mixed-A and mixed-B pathways, respectively. The reaction free energies are noticeably lower when explicit solvation is included, indicative of its importance. For the alternating mechanism, not only does the reaction Gibbs free energy but also the PDS differs between the two solvation models (Figure S4b). Using only implicit solvation, the PDS is the fourth protonation plus reduction step from ${ }^{*} \mathrm{NHNH}_{2}$ to ${ }^{*} \mathrm{NH}_{2} \mathrm{NH}_{2}$ (step 5) with a reaction free energy of $0.81 \mathrm{eV}$ whereas the implicit + explicit solvation model predicts the PDS to be the second protonation plus reduction step, i.e., ${ }^{*} \mathrm{NNH}$ to ${ }^{*} \mathrm{NHNH}$ (step 3) with a reaction free energy of $0.84 \mathrm{eV}$. The second largest reaction free energy step is the second protonation plus reduction when using only implicit solvation (step 3, reaction Gibbs free energy of $0.68 \mathrm{eV}$ ) and the fourth protonation plus reduction for implicit + explicit solvation (step 5, reaction Gibbs free energy of $0.61 \mathrm{eV}$ ). Given that the differences between the PDS and the next largest reaction free energies in each model are larger than $0.1 \mathrm{eV}$ (larger than the expected uncertainty in our predictions) we contend that the predicted PDSs in Figure S4b are unique within each model. Because the mixed implicit + explicit solvation model not only changed 
the predicted reaction free energies but also changed the PDS, we report only the more accurate energies from the mixed implicit + explicit solvation model in the main text.
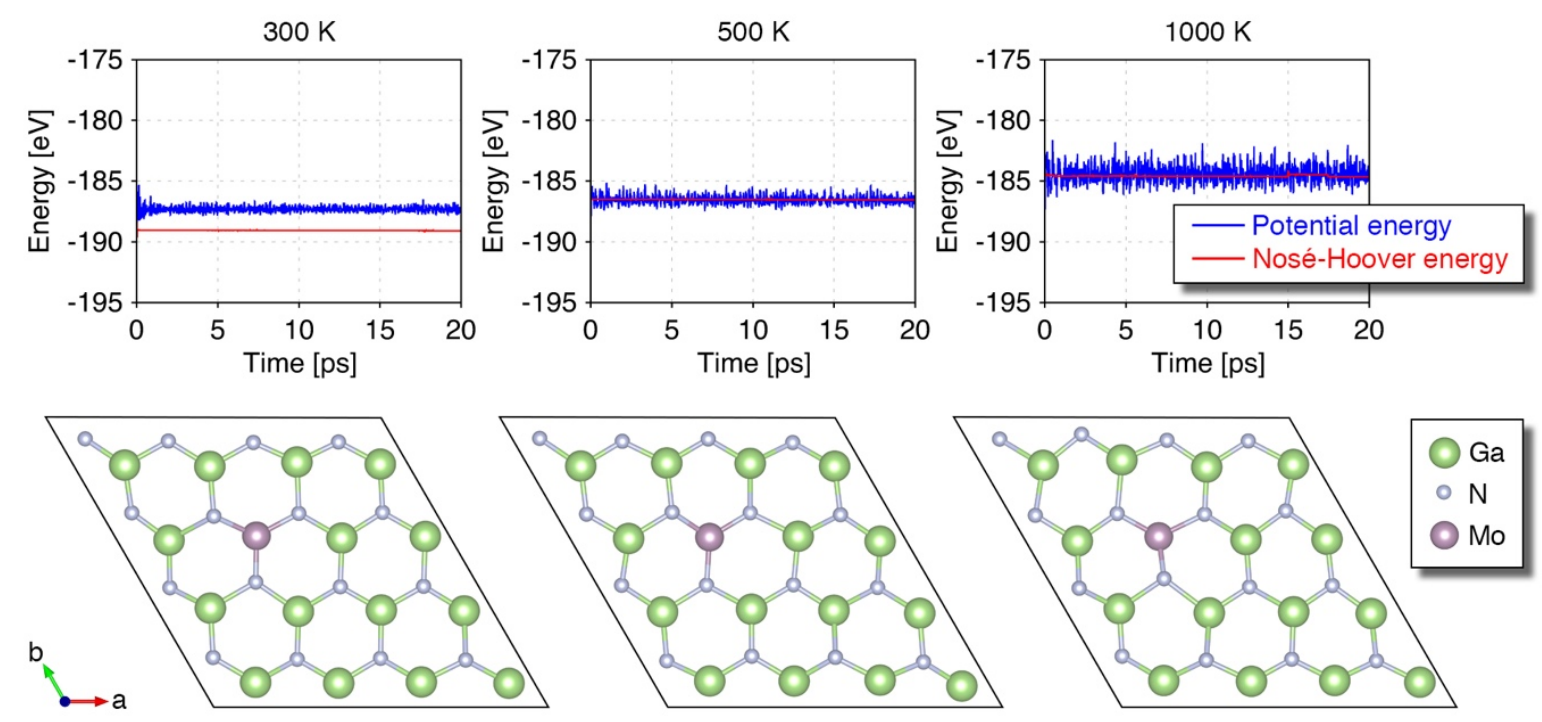

Figure S5. Top: Potential energies and Nosé-Hoover energies as a function of time at temperatures of 300, 500, and $1000 \mathrm{~K}$ for the Mo@g-GaN monolayer. Bottom: Snapshots of the Mo@g-GaN monolayer at the end of the DFT-PBE-D3 molecular dynamics simulation at different temperatures. The simulations were performed with an implicit solvation model. 

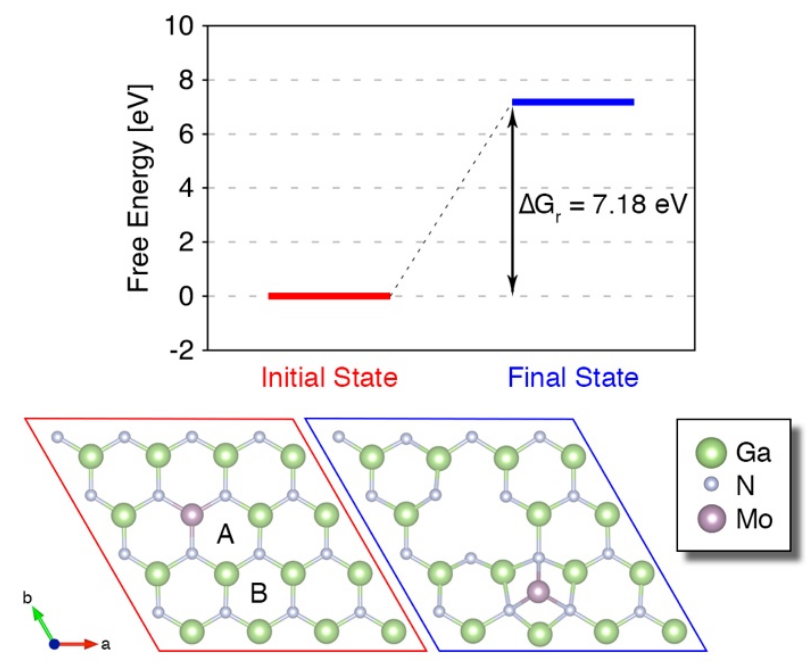

Figure S6. Implicitly solvated DFT-PBE-D3 Gibbs free energy change for diffusion of a single Mo atom from the anchoring site to a neighboring hollow site (top) and the optimized geometries of the associated initial (red) and final (site B; blue) states.

To study diffusion of a single Mo atom, we investigated two different sites for the Mo atom to diffuse to: site A (adjacent hollow) and site B (neighboring hollow) as shown in Figure S6. The Mo atom in site A is not stable (it immediately returns to the initial state during optimization); we therefore only display the Gibbs free energy profile for the diffusion for a single Mo atom to the neighboring hollow site (site B) in Figure S6. 
(a)

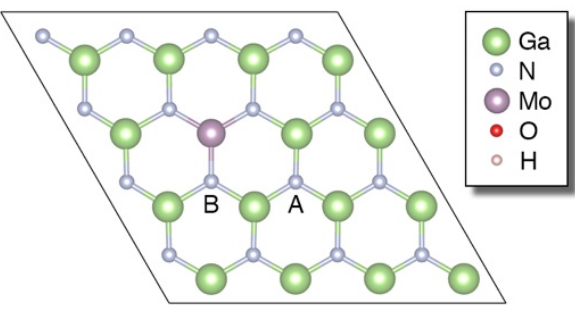

(c)

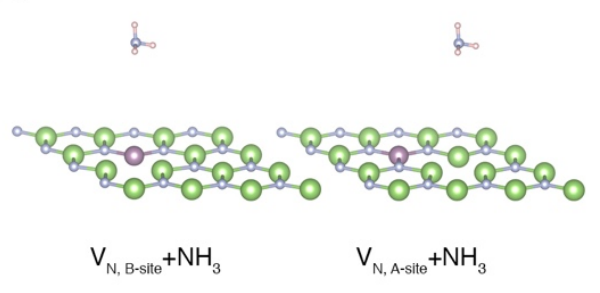

(e)

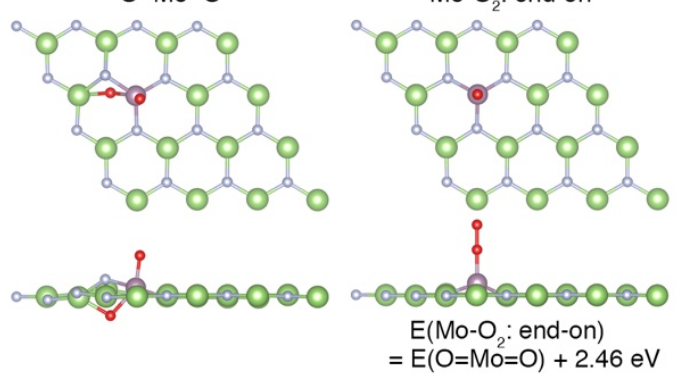

(d) (b)
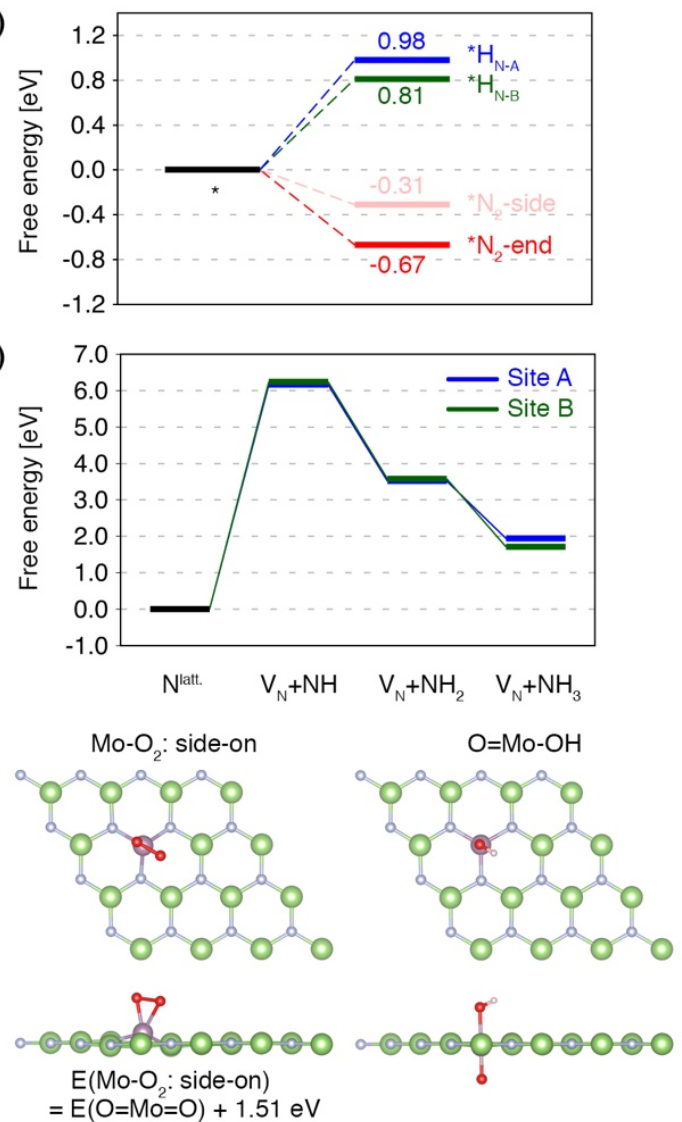
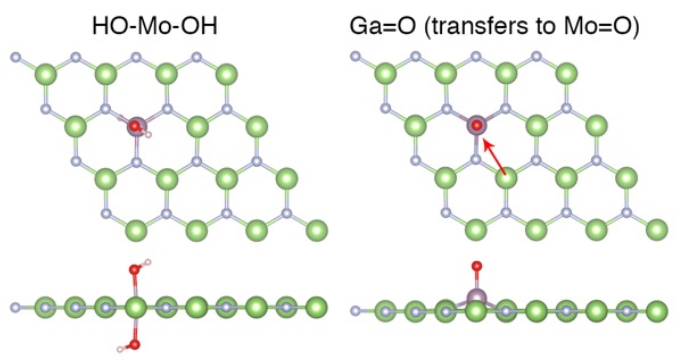

i
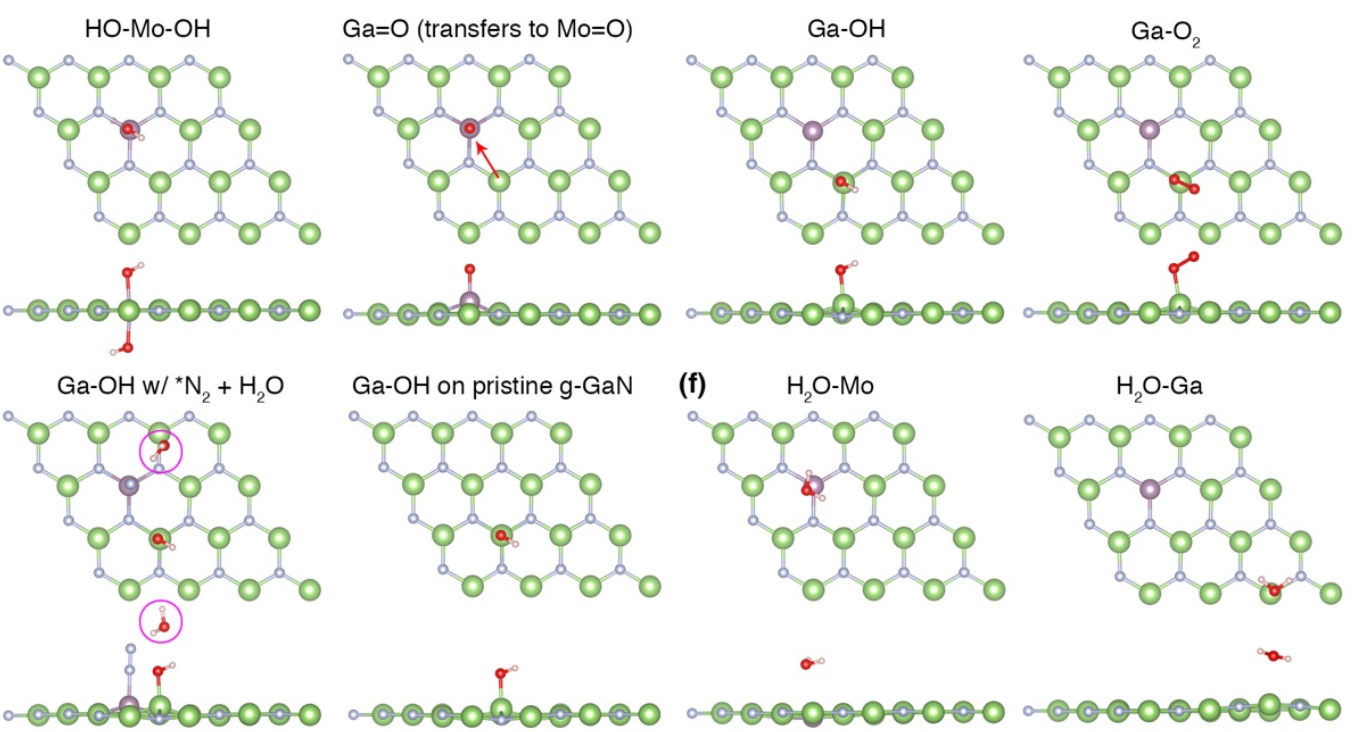

Figure S7. (a) $\mathrm{N}^{\text {latt. }}$ sites A (second-nearest-neighbor $\mathrm{N}$ to Mo) and B (nearest-neighbor N to Mo) in the Mo@g-GaN monolayer. (b) Adsorption Gibbs free energy of H on $\mathrm{N}^{\text {latt. }}$ sites A and B, and of $\mathrm{N}_{2}$ (via end-on and side-on configurations) on Mo in the Mo@g-GaN monolayer at $0 \mathrm{~V}$ vs. the RHE. (c) Optimized geometries (side views) of $\mathrm{V}_{\mathrm{N}}+\mathrm{NH}_{3}$ removing lattice $\mathrm{N}$ atoms from $\mathrm{A}$ - and B-sites. (d) Gibbs free energy profile at $0 \mathrm{~V}$ vs. the RHE for the reaction of $\mathrm{N}^{\text {latt. }}$ (A or $\left.\mathrm{B}\right)+n\left(\mathrm{H}^{+}\right.$ $\left.+\mathrm{e}^{-}\right) \rightarrow \mathrm{V}_{\mathrm{N}}(\mathrm{A}$ or $\mathrm{B})+\mathrm{NH}_{n}$, where $\mathrm{V}_{\mathrm{N}}$ represents the substrate with a $\mathrm{N}$ vacancy at either an $\mathrm{A}$ - or 
B-site, $n$ is the number of $\mathrm{H}$ added onto $\mathrm{N}$ to form $\mathrm{NH}_{n}$. In these reactions, $\mathrm{NH}_{n}$ molecules are $\sim 6$ $\AA$ away from the surface. (e) Optimized geometries (top and side views) of $\mathrm{O}=\mathrm{Mo}=\mathrm{O} @ \mathrm{~g}-\mathrm{GaN}$, Mo-O $\mathrm{O}_{2} @ g-G a N$ via end-on and side-on configurations, O=Mo-(OH)@g-GaN, (OH)-Mo$(\mathrm{OH}) @ g-G a N, \mathrm{Mo}=\mathrm{O} @ g-G a N$, Ga-(OH) on Mo@g-GaN, Ga-O 2 on Mo@g-GaN, Ga-(OH) on Mo@g-GaN with adsorbed $\mathrm{N}_{2}$ (with one explicit water, outlined by a pink circle) at the adjacent Mo site, and $\mathrm{Ga}-(\mathrm{OH})$ on pristine g-GaN monolayers. Note that $\mathrm{Ga}=\mathrm{O}$ on Mo@g-GaN is not stable; the $\mathrm{O}$ moves to the adjacent Mo site during geometry optimization to form $\mathrm{Mo}=\mathrm{O} @ \mathrm{~g}-\mathrm{GaN}$ (as shown). The red arrow in the top view of $\mathrm{Mo}=\mathrm{O} @ \mathrm{~g}-\mathrm{GaN}$ illustrates the $\mathrm{O}$ transfer path from $\mathrm{Ga}=\mathrm{O}$ on Mo@g-GaN to Mo=O@g-GaN. (f) Optimized geometries (top and side views) of a water molecule near the lattice Mo ( 2.9 $\AA$ away) and Ga ( 2.7 $\AA$ away) sites on the Mo@g-GaN. All calculations utilized DFT-PBE-D3 with implicit solvation only (except for explicit solvation for $\mathrm{N}_{2}$ ). We included one explicit water molecule for the adsorbed $\mathrm{N}_{2}$ in order to be consistent with the evaluation of the thermodynamics of $\mathrm{N}_{2}$ adsorption (Section 3.3 in the main text).

\section{SUPPLEMENTARY NOTE 1}

We considered $\mathrm{O}_{2}$ adsorbed in both monodentate (i.e., end-on, vertical and bent) and bidentate (i.e., side-on, horizontal) configurations. Bent $\mathrm{O}_{2}$ bound to Mo was not stable, as it relaxed to the bidentate configuration, whereas bent $\mathrm{O}_{2}$ bound to $\mathrm{Ga}$ was, with both the vertical and bidentate configurations relaxing to the bent $\mathrm{O}_{2}$ configuration during geometry optimization (Figure S7e). We considered different spin states of the $\mathrm{O}$ atoms in the monodentate configurations by specifying different initial total magnetic moments for the two $\mathrm{O}$ atoms, namely, with $(0,1)$ or $(1,1)$ number of unpaired electrons ( $\mathrm{O}$ attached to $\mathrm{Mo} / \mathrm{Ga}$, outer $\mathrm{O}$ ). The calculated total internal energies (without zero-point energies) of $\mathrm{Mo}-\mathrm{O}_{2}$ in end-on and side-on configurations are respectively 2.46 and $1.51 \mathrm{eV}$ higher than that of $\mathrm{O}=\mathrm{Mo}=\mathrm{O}$, while the bent $\mathrm{Ga}-\mathrm{O}_{2}$ species was highly unstable with a very positive (+2.98 eV) adsorption energy at zero applied potential (see main text for details).

For end-on $\mathrm{Mo}-\mathrm{O}_{2}$, the outer $\mathrm{O}$ atom relaxes to a projected magnetization of 0 , with net Bader charges of $-0.51 e$ and $-0.79 e$ for the outer $\mathrm{O}$ atom and the $\mathrm{O}$ atom bonded to Mo, respectively, for a total of $-1.30 e$, implying a reduction of the dioxygen molecule. The net Bader charges on the 
bidentate Mo- $\mathrm{O}_{2}$ are $-0.65 e$ and $-0.72 e$, for a total of $-1.37 e$. Bent $\mathrm{Ga}-\mathrm{O}_{2}$ has net Bader charges of $-0.47 e$ and $-0.72 e$ respectively for the outer $\mathrm{O}$ atom and the $\mathrm{O}$ atom attached to the lattice $\mathrm{Ga}$ atom (-1.19 $e$ total), also implying reduction of dioxygen, although slightly less than on Mo.

Because of the relative instability of any adsorbed dioxygen species, we did not evaluate their Gibbs free energies; we only evaluated the Gibbs free energies of the most stable configuration $(\mathrm{O}=\mathrm{Mo}=\mathrm{O})$, along with the less oxidized forms of the Mo site derived from this most stable configuration, namely $\mathrm{O}=\mathrm{Mo}-\mathrm{OH}$ and $\mathrm{HO}-\mathrm{Mo}-\mathrm{OH}$. The potential dependence of the oxidation free energies $\left(\Delta G_{o x}\right)$ of a generic reaction:

$$
\mathrm{Mo@g-GaN}+n \mathrm{H}_{2} \mathrm{O} \rightarrow \mathrm{Mo}\left(\mathrm{O}_{n} \mathrm{H}_{m}\right) @ g-G a N+(2 n-m)\left(\mathrm{H}^{+}+\mathrm{e}^{-}\right)
$$

was calculated using the Nernst equation for an oxidation half-reaction:

$$
\Delta G_{o x}=\Delta G_{o x}^{\circ}-(2 n-m) q U_{\mathrm{vs} . \mathrm{RHE}}
$$

where $\Delta G^{\circ}{ }_{o x}$ is the standard oxidation free energy with $G_{\mathrm{H}^{+}+\mathrm{e}^{-}}^{\circ}=(1 / 2) G_{\mathrm{H}_{2}}^{\circ}, q=1$ is the electron charge, and $U_{\mathrm{vs} \text {. RHE }}$ is the applied potential vs. the reversible hydrogen electrode (RHE) (see also "COMPUTATIONAL DETAILS AND MODELS" in the main text for further explanation).

Finally, a water molecule will not adsorb on either the Mo or the Ga atom in the substrate (e.g., Figure S7f), instead moving $\sim 2.9$ and $\sim 2.7 \AA$ away from the lattice Mo and Ga, respectively, into solution. The adsorption Gibbs free energy of $\mathrm{H}_{2} \mathrm{O}(l)$ is computed using the equation:

$$
\Delta G_{\mathrm{H}_{2} \mathrm{O}(l)-a d s}=G\left(\mathrm{H}_{2} \mathrm{O}-\mathrm{Mo} \text { or } \mathrm{H}_{2} \mathrm{O}-\mathrm{Ga} \text { on Mo@g-GaN }\right)-G\left(\mathrm{H}_{2} \mathrm{O}(l)\right)-G(\mathrm{Mo} @ g-G a N)
$$


where $G\left(\mathrm{H}_{2} \mathrm{O}-\mathrm{Mo}\right.$ or $\mathrm{H}_{2} \mathrm{O}-\mathrm{Ga}$ on $\left.\mathrm{Mo@g-GaN}\right), G\left(\mathrm{H}_{2} \mathrm{O}(l)\right)$, and $G(\mathrm{Mo} @ g-\mathrm{GaN})$ are respectively the Gibbs free energies of Mo@g-GaN with a $\mathrm{H}_{2} \mathrm{O}$ molecule near either the Mo or a Ga site, a solvated water molecule, and the adsorbate-free Mo@g-GaN monolayer. Details on how to calculate each of the Gibbs free energy terms are discussed in the main text (COMPUTATIONAL DETAILS AND MODELS). We predict $\Delta G_{\mathrm{H}_{2} \mathrm{O}(l)-a d s}$ at the Mo and Ga sites to be +0.23 and $+0.10 \mathrm{eV}$, respectively. The propensity of water to relax relatively far away from the surface and the positive free energies for water adsorption near Mo or Ga imply hydrophobicity of the surface. This hydrophobicity may further favor the approach of non-polar molecules, such as $\mathrm{N}_{2}$, over water, possibly further suppressing oxidative adsorption of water (reactions described above) and possibly even inhibiting the HER. 

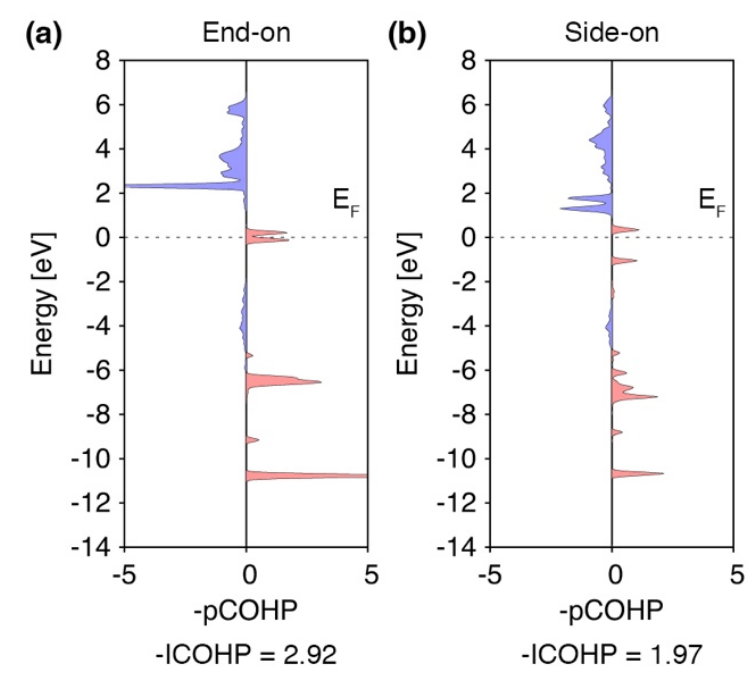

Figure S8. Projected crystal orbital Hamilton population (-pCOHP) analysis between the Mo atom and the nearest $\mathrm{N}$ atom in $\mathrm{N}_{2}$ adsorbed (a) end-on and (b) side-on to the Mo@g-GaN monolayer, for the spin-down component, based on DFT-PBE-D3 calculations with a mixed implicit + explicit solvation model. The Fermi level is set to the reference energy of $0 \mathrm{eV}$ in the pCOHP analysis. Red and blue shaded regions denote bonding and anti-bonding contributions, respectively. The negative of the integrated COHP (-ICOHP) values are given under each panel. 

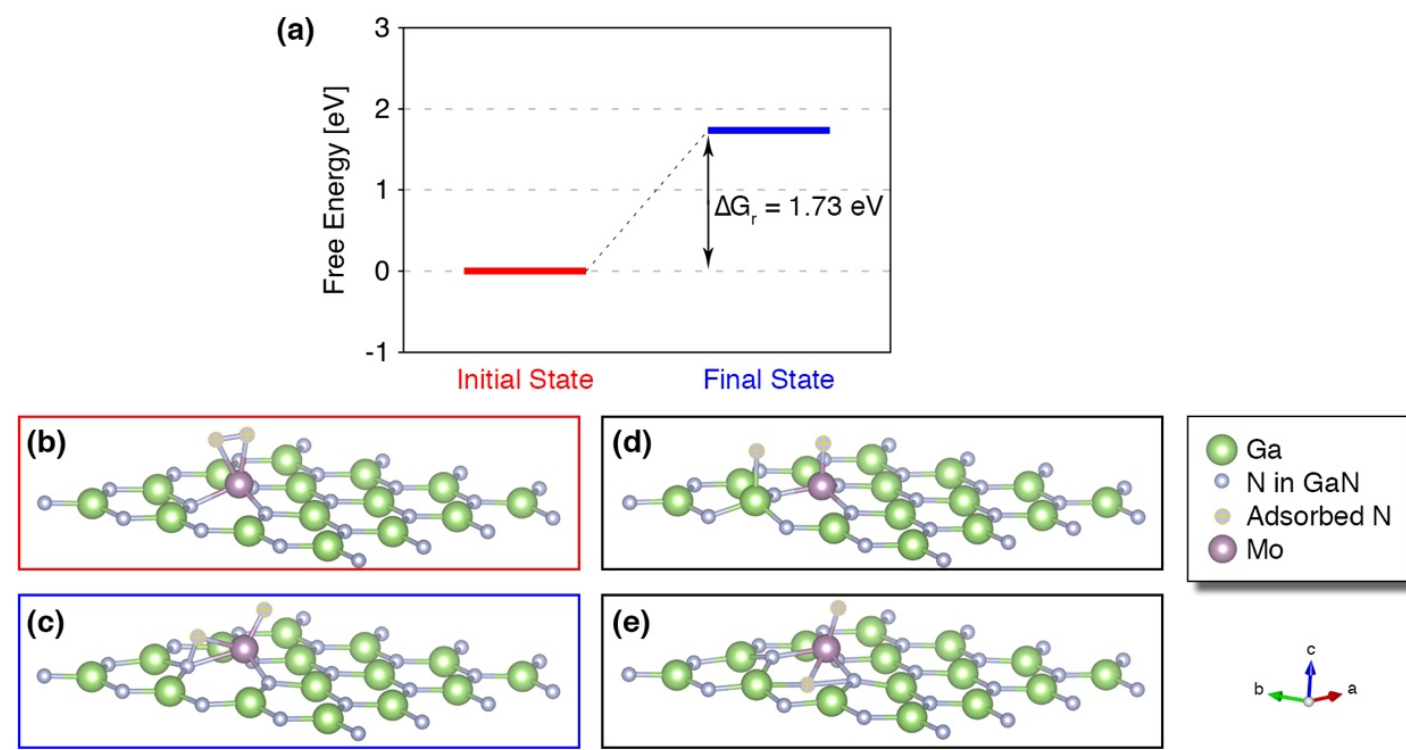

Figure S9. (a) Gibbs free energy profile of the dissociation of adsorbed $\mathrm{N}_{2}$ (side-on configuration) into two separate $\mathrm{N}$ atoms on the Mo@g-GaN monolayer, based on DFT-PBE-D3 calculations with implicit solvation only. Optimized geometries of the initial (b) and final states where the adsorbed ${ }^{*} \mathrm{~N}_{2}$ dissociates to the adjacent (c) lattice $\mathrm{N}$ atom, (d) Ga atom, and (e) hollow site on the Mo@g-GaN monolayer.

To investigate the dissociation of side-on adsorbed ${ }^{*} \mathrm{~N}_{2}$, three different adjacent adsorption sites for the $\mathrm{N}$ atom were considered: a lattice $\mathrm{N}$ atom (Figure S8c), a Ga atom (Figure S8d), and a hollow site (Figure S8e) on the Mo@g-GaN monolayer. $\mathrm{N}_{2}$ dissociative adsorption onto the adjacent $\mathrm{Ga}$ atom and hollow site are less favorable than onto an adjacent lattice $\mathrm{N}$ atom, with total energies 2.14 and $2.85 \mathrm{eV}$ higher than the latter case. Because the energetics of the $\mathrm{N}$ on the adjacent Ga atom and hollow site were so unfavorable, they were not verified further as to whether they were local minima. Therefore, we only show the Gibbs free energy profile of the dissociation of the side-on adsorbed ${ }^{*} \mathrm{~N}_{2}$ onto the adjacent lattice $\mathrm{N}$ atom of the Mo@g-GaN monolayer in Figure S8a. 


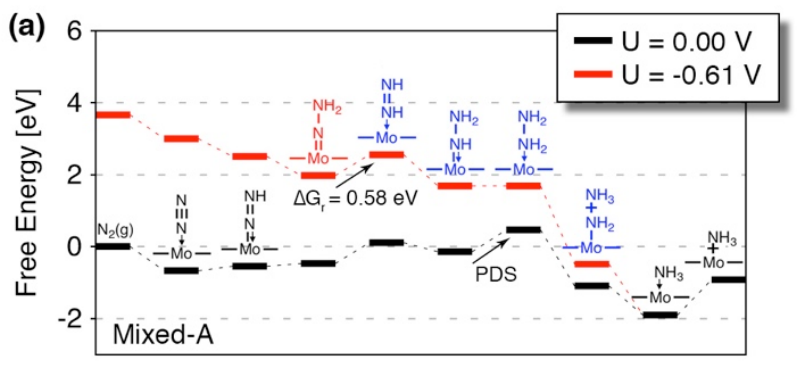

Reaction Pathway

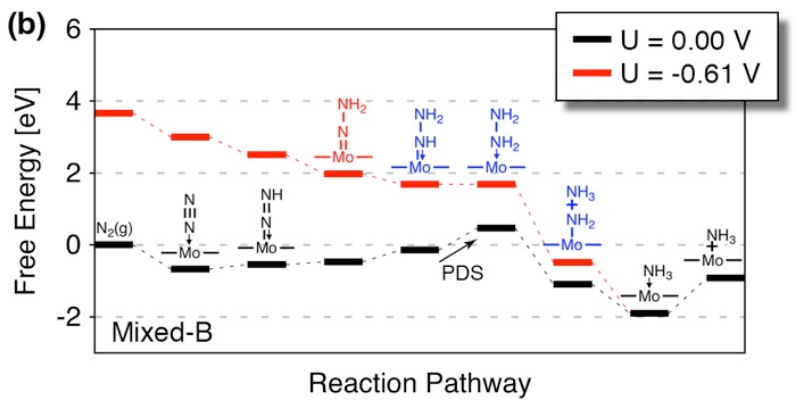

Figure S10. Gibbs free energy diagrams for the NRR on the Mo@g-GaN monolayer via (a) mixedA and (b) mixed-B mechanisms at zero and at an applied potential equal to the onset potential, based on DFT-PBE-D3 calculations with a mixed implicit + explicit solvation model. The PDS is labeled in each Gibbs free energy diagram. Note the remaining endoergic (isomerization) step in (a) with $\Delta \mathrm{G}_{\mathrm{r}}=0.58 \mathrm{eV}$ does not depend on applied potential. 


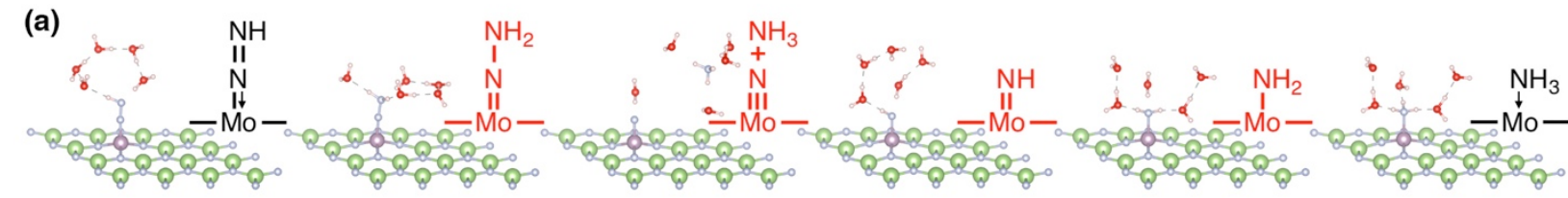

(b)

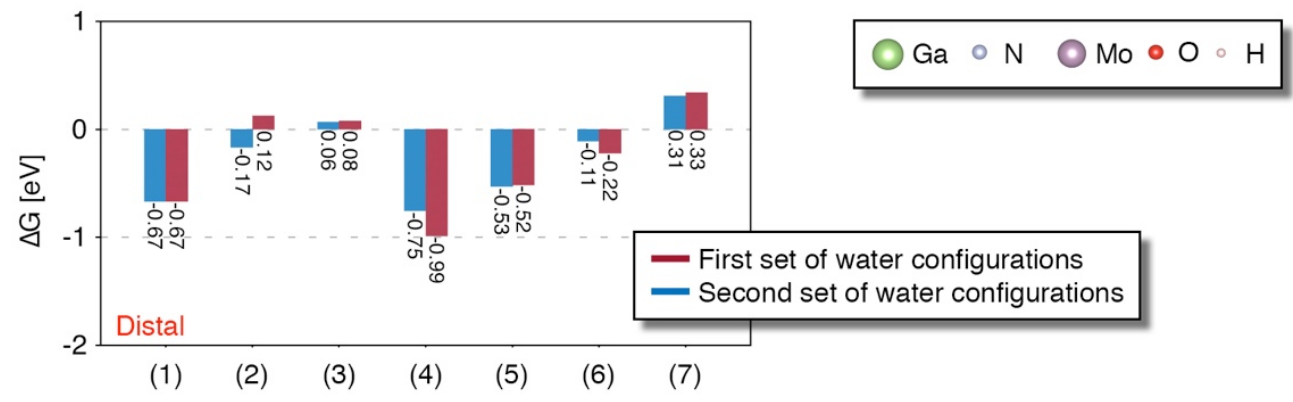

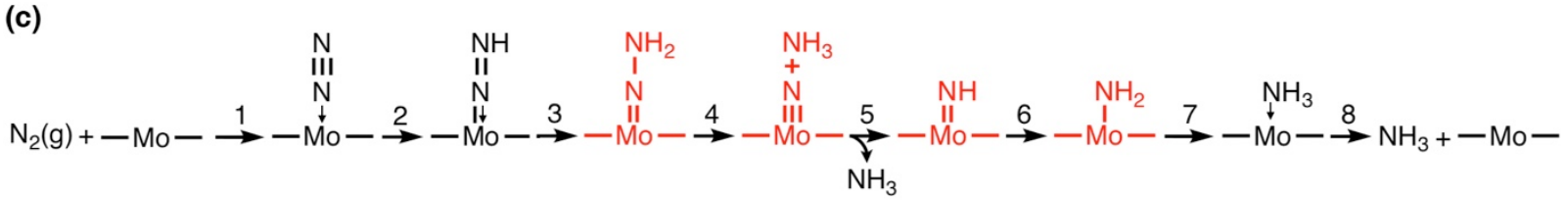

Figure S11. (a) Optimized geometries of the intermediates along the distal pathway from DFTPBE-D3 with the mixed implicit + explicit solvation model for the second set of water configurations. Note that the water structures for Mo $\leftarrow \mathrm{N} \equiv \mathrm{N}$ (not shown) and $\mathrm{Mo} \equiv \mathrm{N}+\mathrm{NH}_{3}$ were not modified because all of their solvating water molecules are in the first solvation shell. (b) Reaction Gibbs free energies along the distal pathway for both the first (red) and second (blue) set of water configurations. The structure labels in (a) and step indices on the horizontal axis in (b) correspond to the labels and step numbers in (c). 


\section{SUPPLEMENTARY NOTE 2}

To determine the kinetic barrier of the potential-determining step, i.e., ${ }^{*} \mathrm{NH}_{2}$ to ${ }^{*} \mathrm{NH}_{3}$, we followed the method proposed by Chan and Norskøv. ${ }^{6-7}$ The Gibbs free energy changes from state A to state B (including both reaction Gibbs free energy and activation Gibbs free energy) at the potential corresponding to the work function of state A and of state B, are computed as:

$$
\begin{aligned}
& G_{B}\left(\Phi_{A}\right)-G_{A}\left(\Phi_{A}\right)=G_{B}\left(\Phi_{B}\right)-G_{A}\left(\Phi_{A}\right)+\frac{\left(q_{B}-q_{A}\right)\left(\Phi_{B}-\Phi_{A}\right)}{2} \\
& G_{B}\left(\Phi_{B}\right)-G_{A}\left(\Phi_{B}\right)=G_{B}\left(\Phi_{B}\right)-G_{A}\left(\Phi_{A}\right)-\frac{\left(q_{B}-q_{A}\right)\left(\Phi_{B}-\Phi_{A}\right)}{2}
\end{aligned}
$$

where $G$ is the Gibbs free energy, $\Phi$ is the potential, and $q$ is the excess charge (electron). The excess electron $q$ is calculated via Bader charge analysis, which is a real-space charge partitioning scheme (Table S4). ${ }^{8-10}$

Table S4. Calculated excess charge $q$ of the Mo@g-GaN and the adsorbate ( ${ }^{*} \mathrm{NH}_{2}$ for IS and TS or ${ }^{*} \mathrm{NH}_{3}$ for FS) from the stationary and transition state structures for the PDS (step 7) of the distal pathway via the Bader charge analysis. ${ }^{a}$

\begin{tabular}{cccc}
\hline \hline Systems & IS & TS & FS \\
\hline$q$ value $(e)$ & 0.848 & 0.792 & 0.049 \\
\hline \hline
\end{tabular}

$\overline{\bar{a} \text { The calculated } q \text { s are used to evaluate the work function-dependence of the free energies of }}$ reaction and barriers of the potential-dependent PDS.

In their original formulation, the above two equations establish the dependence of the reaction/activation Gibbs free energy on $\Phi$, which is the work function of the surface, which by definition is measured under vacuum conditions and is computed from the electrostatic potential difference between vacuum and the center of periodic slab model representing the surface. Here, because an implicit solvation model is used, calculation of the work function will require an additional explicit vacuum region in the simulation, which is currently not implemented in the 
software we employ. Alternatively, knowledge of the electronic level of the implicit solvent with respect to the vacuum ( $\left.\Phi_{\text {implicit solvation }}\right)$ could be used. However, instead of identifying the work function of the implicit solvent, we used directly the average electrostatic potential of the slab center relative to the electrostatic potential of the middle region of the implicit solvent (see Figure S3 for an example). This is valid because $\Phi_{\text {implicit solvation }}$ is simply a constant for a given implicit solvation model, and one can easily show that the dependence of the Gibb's free energy of reaction or activation with the potential measured relative to the implicit solvent $(\Delta \Phi=\Phi-$ $\left.\Phi_{\text {implicit solvation }}\right)$ is the same as in $\Phi$ :

$$
\begin{aligned}
G_{B}\left(\Delta \Phi_{A}\right)-G_{A} & \left(\Delta \Phi_{A}\right)=G_{B}\left(\Delta \Phi_{B}\right)-G_{A}\left(\Delta \Phi_{A}\right)+\frac{\left(q_{B}-q_{A}\right)\left(\Delta \Phi_{B}-\Delta \Phi_{A}\right)}{2} \\
& =G_{B}\left(\Delta \Phi_{B}\right)-G_{A}\left(\Delta \Phi_{A}\right) \\
& +\frac{\left(q_{B}-q_{A}\right)\left(\Phi_{B}-\Phi_{\text {implicit solvation }}-\Phi_{A}+\Phi_{\text {implicit solvation }}\right)}{2} \\
& =G_{B}\left(\Delta \Phi_{B}\right)-G_{A}\left(\Delta \Phi_{A}\right)+\frac{\left(q_{B}-q_{A}\right)\left(\Phi_{B}-\Phi_{A}\right)}{2}
\end{aligned}
$$

Moreover, note that from the original equations, for any $\Phi$, the pertinent linear equation is of the form

$$
G_{B}(\Phi)-G_{A}(\Phi)=-\left(q_{B}-q_{A}\right)(\Phi)+\left[G_{B}\left(\Phi_{B}\right)-G_{A}\left(\Phi_{A}\right)+\frac{\left(q_{B}-q_{A}\right)\left(\Phi_{B}+\Phi_{A}\right)}{2}\right]
$$

in which $-\left(q_{B}-q_{A}\right)$ is the slope and the whole second term in square brackets is the constant yintercept. Therefore, for any $\Phi$, say $\Phi=\Delta \Phi_{A}=\Phi_{A}-\Phi_{\text {implicit solvation }}$ 


$$
\begin{aligned}
G_{B}\left(\Delta \Phi_{A}\right)-G_{A} & \left(\Delta \Phi_{A}\right)=-\left(q_{B}-q_{A}\right)\left(\Delta \Phi_{A}\right)+\left[G_{B}\left(\Phi_{B}\right)-G_{A}\left(\Phi_{A}\right)+\frac{\left(q_{B}-q_{A}\right)\left(\Phi_{B}+\Phi_{A}\right)}{2}\right] \\
& =\left(q_{B}-q_{A}\right)\left(\Phi_{\text {implicit solvation }}\right)+\left[G_{B}\left(\Phi_{B}\right)-G_{A}\left(\Phi_{A}\right)+\frac{\left(q_{B}-q_{A}\right)\left(\Phi_{B}+\Phi_{A}\right)}{2}\right] \\
& -\left(q_{B}-q_{A}\right) \Phi_{A}
\end{aligned}
$$

note however that

$$
\begin{aligned}
G_{B}\left(\Phi_{B}\right)-G_{A}\left(\Phi_{A}\right)+\frac{\left(q_{B}-q_{A}\right)\left(\Phi_{B}+\Phi_{A}\right)}{2}-\left(q_{B}-q_{A}\right) \Phi_{A} \\
\quad=G_{B}\left(\Phi_{B}\right)-G_{A}\left(\Phi_{A}\right)+\frac{\left(q_{B}-q_{A}\right)\left(\Phi_{B}-\Phi_{A}\right)}{2}=G_{B}\left(\Phi_{A}\right)-G_{A}\left(\Phi_{A}\right)
\end{aligned}
$$

therefore

$$
G_{B}\left(\Delta \Phi_{A}\right)-G_{A}\left(\Delta \Phi_{A}\right)=G_{B}\left(\Phi_{A}\right)-G_{A}\left(\Phi_{A}\right)+\left(q_{B}-q_{A}\right)\left(\Phi_{\text {implicit solvation }}\right)
$$

or

$$
G_{B}\left(\Phi_{A}\right)-G_{A}\left(\Phi_{A}\right)=G_{B}\left(\Delta \Phi_{A}\right)-G_{A}\left(\Delta \Phi_{A}\right)-\left(q_{B}-q_{A}\right)\left(\Phi_{\text {implicit solvation }}\right)
$$

where $-\left(q_{B}-q_{A}\right)\left(\Phi_{\text {implicit solvation }}\right)$ is a constant, in fact for any $\Phi$. Therefore, the use of $\Delta \Phi$ instead of $\Phi$ can also be thought of as a simple shift by $+\Phi_{\text {implicit solvation }}$ along the horizontal axis of the plot of $\Delta G_{r}$ or $\Delta G^{\ddagger}$ as a function of $\Phi$. 


\section{REFERENCES}

(1) Neugebauer, J.; Scheffler, M., Adsorbate-Substrate and Adsorbate-Adsorbate Interactions of Na and K Adlayers on Al(111). Phys. Rev. B 1992, 46, 16067-16080.

(2) Kresse, G.; Furthmüller, J., Efficiency of Ab-Initio Total Energy Calculations for Metals and Semiconductors Using a Plane-Wave Basis Set. Comput. Mater. Sci. 1996, 6, 15-50.

(3) Kresse, G.; Furthmüller, J., Efficient Iterative Schemes for Ab Initio Total-Energy Calculations Using a Plane-Wave Basis Set. Phys. Rev. B 1996, 54, 11169-11186.

(4) Mathew, K.; Sundararaman, R.; Letchworth-Weaver, K.; Arias, T.; Hennig, R. G., Implicit Solvation Model for Density-Functional Study of Nanocrystal Surfaces and Reaction Pathways. J. Chem. Phys. 2014, 140, 084106.

(5) Mathew, K.; Hennig, R., Implicit Self-Consistent Description of Electrolyte in Plane-Wave Density-Functional Theory. arXiv preprint arXiv:1601.03346 2016.

(6) Chan, K.; Nørskov, J. K., Electrochemical Barriers Made Simple. J. Phys. Chem. Lett. 2015, 6, 2663-2668.

(7) Chan, K.; Nørskov, J. K., Potential Dependence of Electrochemical Barriers from Ab Initio Calculations. J. Phys. Chem. Lett. 2016, 7, 1686-1690.

(8) Henkelman, G.; Arnaldsson, A.; Jónsson, H., A Fast and Robust Algorithm for Bader Decomposition of Charge Density. Comput. Mater. Sci. 2006, 36, 354-360.

(9) Sanville, E.; Kenny, S. D.; Smith, R.; Henkelman, G., Improved Grid-Based Algorithm for Bader Charge Allocation. J. Comput. Chem. 2007, 28, 899-908.

(10) Tang, W.; Sanville, E.; Henkelman, G., A Grid-Based Bader Analysis Algorithm without Lattice Bias. J. Phys.: Condens. Matter 2009, 21, 084204. 